\title{
The need for multidisciplinary health research
}

The realisation of the goal of health for all requires a concerted action of many sectors, including health, agriculture, education and sociology. Thus, there is a need to carry out multidisciplinary research to address emerging health problems in Sri Lanka and the South Asian region. The factors that contribute to the change in patterns of disease, such as urbanisation, industrialisation, globalisation, changes in environment, population growth and increase in the median age of the population, require a change from conventional discipline based thinking to interdisciplinary approaches.

Achieving food security ie provision of adequate supplies of safe, nutrient-rich foods to meet dietary needs, at both national and household level is essential to ensure the health and wellbeing of our people. Ongoing research to develop newer technologies to augment food production, identify drought and pest resistant varieties, and minimize post harvest losses of cereals, pulses, fruits and vegetables need to be strengthened. Micronutrient deficiencies, specially, those of iron, vitamin $A$, folic acid and zinc are important problems in many Asian countries including Sri Lanka. The data available on the prevalence of these deficiencies in Sri Lanka provides a basis for planning research on genetic enhancement of nutritional quality and biofortification to increase the nutritive value of commonly available foods. For example, research to develop varieties of rice rich in the micronutrients, iron, zinc, provitamin $A$ and folic acid and low in phytic acid is an essential step in preventing micronutrient deficiencies. There should be a greater interaction between health professionals and researchers in food and agriculture to identify areas for research. Further, multidisciplinary studies on bioavailability of micronutrients such as iron and zinc in local diets are necessary to provide information for development of nutrition education materials.
Research on effects of environmental factors on health has received only limited attention. The alteration of the trace element content of soil and water and its effect on health, for example a study of the factors causing increase in concentration of fluoride in water in certain areas is necessary to prevent the adverse effects of fluorosis. Interdisciplinary research is necessary to study the relationship between the dramatic increase in incidence of Asthma and environmental pollution. A multidisciplinary and global collaboration was the key to discovering the causative agent for Severe Acute Respiratory Syndrome (SARS) and also for controlling the spread of SARS among health care workers. Controlling emerging infections such as Avian flu, also require inputs not only from health researchers, but also from researchers in veterinary and environmental sciences. The incidence of diet-related noncommunicable diseases such as, hypertension, diabetes, cardiovascular disease has increased dramatically in Sri Lanka and other Asian countries. There is emerging evidence that Asians have a greater genetic predisposition to insulin resistance than Caucasians even when the body size is small. Research on gene-nutrient interactions - nutrigenetics/nutrigenomics is likely to shed light on this aspect.

Research carried out in Sri Lanka is often presented at scientific meetings, but publications are limited. Further, research in other disciplines which have a bearing on health is either published in highly specialised journals, or not published at all, and often does not reach health professionals. Similarly, research on common health problems must be accessible to researchers in other scientific disciplines in order to plan out research on health related issues. Thus there is a need to disseminate this information through multidisciplinary peer-reviewed journals. Dissemination of research findings is as important as carrying out research. Greater emphasis to multidisciplinary research is likely to have a significant positive impact on the health of our people. 